\title{
Nurses' Perceptions of Patient Safety in Community Mental Health Settings: A Qualitative Study
}

\author{
Rakel Sundin'1, Annika Nilsson'2,3, Rebecka Waage-Andrée4, Catrine Björn ${ }^{3,5,6 *}$ \\ ${ }^{1}$ Caring Sciences, Municipality in Söderhamn, Sweden \\ ${ }^{2}$ Faculty of Health and Occupational Studies, Department of Health and Caring Sciences, University of Gävle, \\ Gävle, Sweden \\ ${ }^{3}$ Department of Public Health and Caring Sciences, Section of Caring Sciences, Uppsala University, Uppsala, \\ Sweden \\ ${ }^{4}$ Department of Endocrinology, Gävle Hospital, Gävle, Sweden \\ ${ }^{5}$ Centre for Research \& Development, Uppsala University/Region Gävleborg, Gävle, Sweden \\ ${ }^{6}$ Department of Occupational and Environmental Medicine, Uppsala University Hospital, Uppsala, Sweden \\ Email: catrine.bjorn@regiongavleborg.se
}

Received 18 March 2015; accepted 23 April 2015; published 28 April 2015

Copyright (C) 2015 by authors and Scientific Research Publishing Inc.

This work is licensed under the Creative Commons Attribution International License (CC BY).

http://creativecommons.org/licenses/by/4.0/

\section{(c) (i) Open Access}

\section{Abstract}

Mental health care has moved from hospital settings to community mental health settings, and there is a need to explore the perceptions of patient safety among registered nurses working in this field. Patient safety is to include everyone and to be the goal in all aspects of health care. The aim of the study was to explore registered nurses' perceptions of patient safety in community mental health settings for people with serious mental illness. The study was qualitative and descriptive in nature and interviews were carried out during spring 2012, with seven registered nurses working in community mental health settings for people with serious mental illness in five municipalities in the middle of Sweden. The sampling was purposive and data were analysed using qualitative content analysis. The findings showed that the registered nurses understood patient safety as providing support to people with serious mental illness in regaining and maintaining health through good treatment and respecting self-determination and avoiding coercion. The terms of daily living in small community mental health settings within the a large community health care organization, communication, sufficient knowledge of psychiatric disabilities among people in the residents' network, and national laws and regulations, all had implications for patient safety. The registered nurses perceived patient safety as involving a wide range of issues that

${ }^{*}$ Corresponding author.

How to cite this paper: Sundin, R., Nilsson, A., Waage-Andrée, R. and Björn, C. (2015) Nurses' Perceptions of Patient Safety in Community Mental Health Settings: A Qualitative Study. Open Journal of Nursing, 5, 387-396.

http://dx.doi.org/10.4236/ojn.2015.54042 
in other areas of care are more often discussed in terms of quality of care. Determining the boundaries of patient safety in community mental health settings for people with serious mental illness can be a first step in establishing workable routines that ensure safe patient care.

\title{
Keywords
}

\author{
Community Mental Health Care, Mental Health Nursing, Patient Safety, Qualitative Research
}

\section{Introduction}

During recent decades, mental health care has undergone structural changes intended to bring people with serious mental illness (SMI), such as psychosis or personality disorders, closer to the everyday life of the community. The World Health Organization (WHO) has assumed a leading role in bringing this change about, striving to move mental health care from hospitals to community mental health settings (CMHS). In connection with changes in settings, however, the conditions for maintaining patient safety have also been altered. Patient safety is defined by the WHO as "the absence of preventable harm to a patient during the process of health care". It is to apply to every individual receiving care and to be the goal in all aspects of health care [1]. Following the reform of mental health care, the concept and content of patient safety need to be discussed in the context of this area of care and the partially new factors influencing patient safety. The present study focuses on registered nurses' (RNs) perceptions of patient safety in CMHS for people with SMI.

According to the theory proposed by Leape, patient safety has four main constituent parts: care recipients, care providers, therapeutics, and methods. The theory claims that patient safety is the result of a well-functioning, interactive system rather than of the actions of individuals [2]. The research of patient safety most often has taken this systematic view. But so far, much of the research derives from the perspective of hospital settings offering medical care where issues concerning physical care such as preventing infections, pressure ulcers and post-surgical complications, are the focus [3]. Patient safety in psychiatric care is sparsely investigated. However, a literature review [4] focusing on patient safety in psychiatric inpatient care highlights the diversity of the patient safety concept, showing that neglect in one area may influence other areas, resulting in adverse events. To achieve patient safety organizational management was important, including good leadership, safety practices, good working conditions, and that care was planned according to the patient's preferences [4]. Consequently, patient safety in mental health care appears to involve issues different from those generally discussed in relation to medical care. For example, the stigma connected to SMI entails a risk that patients will not receive adequate care or that the quality of care will be jeopardized due to care attitudes [5] [6]. Research suggests that there is a close connection between patient safety and quality of care, where quality of care can be seen as comprehensive and covering the patient safety concept [3].

The goal with moving the majority of patient care from hospital settings to CMHS has been to empower people with SMI and to create better opportunities for them to participate in community life despite their psychosocial disabilities [7]. Structural changes to achieve this have been taking place in many parts of the world since the middle of the last century, but compared to some of the developing countries, higher income-countries have made more progress in the implementation of the standards from WHO [8] [9]. Some promising research supporting the deinstitutionalization shows that people with SMI living in CMHS reported improved quality of life through friendships and a home like environment, yet there is still a need for more attention to social inclusion among this group [10]. It is also important to have trained personnel who work with people with SMI. In England and Australia several of the nurses working in community mental health care facilities are educated mental health nurses compared to other countries such as USA. In USA people without mental health qualifications perform similar jobs [11] and in Sweden there is also a lack of nurses with specialist education in mental health care [12].

Given the sparse research in the area [5] and to the transition from large hospitals to CMHS, there is a need to explore how RNs perceive patient safety for people with SMI. According to Mwachofi et al. [13], RNs are well equipped to take part in efforts to improve patient safety. In line with this the aim of the study was to explore registered nurses perceptions of patient safety in community mental health settings for people with serious mental illness. 


\section{Materials and Methods}

\subsection{Design}

The study was qualitative and descriptive in nature [14].

\subsection{Sample}

To get in contact with RNs working in the field of CMHS for people with SMI, heads of departments in five municipalities were contacted by telephone and by letter informing them about the study and asking for permission to perform the study. All gave their consent and provided contact information for RNs. Inclusion criteria were that the RN had work experience from CMHS for people with SMI exceeding six months during the past year. Seven out of eight RNs met the inclusion criteria and were sent a letter explaining the aim of the study and an invitation to participate. Within two weeks, the RNs responded to the invitation; all agreed to participate. The two men and five women varied in age from 23 to 65 years. Their length of experience in the field ranged from eight months to 17 years. Half of the RNs had a formal degree in mental health care.

\subsection{Setting}

CMHS for SMI in Sweden offers each person his/her own apartment close to or in immediate connection with a common apartment shared with other residents suffering from SMI. The number of private apartments connected to the common apartment ranged from 5 - 20. In the common apartment enrolled nurses were available round the clock. Each of the RNs was responsible for 2 - 4 CMHS, resulting in a total of approximately 20 residents with SMI per RN. The RNs' offices were located some distance away from the CMHS, ranging from a few blocks up to 25 kilometres. The RNs were responsible for the residents' day-to-day mental and physical health care and for ongoing pharmaceutical therapy carried out by enrolled nurses in CMHS.

\subsection{Data Collection}

Data collection was performed from January through February 2012 using semi-structured interviews. An interview guide [14] was constructed based on national requirements for patient safety, e.g. medically preventing and treating illness, and avoiding injury due to maltreatment or lack of adequate treatment [15]. The interview guide was formed as a palette, Figure 1, and placed openly as common ground during the interviews.

All of the interviews had patient safety as the main topic from which the other questioning perspectives arose. The RNs were asked to give their reflections on patient safety in their field regarding: "The concept of patient safety", "Upholding patient safety", "Improvements possible in patient safety”, and "Good/less good aspects in the area of patient safety". The authors used open-ended questions and probes such as "Would you please elaborate?" or "How do you mean?" to clarify or follow up on answers. The average length of each interview was 30 minutes (23 - 56 minutes). By choice of the RNs the interviews were held in their offices, providing privacy and a quiet environment. The interviews were audio-recorded.

\subsection{Data Analysis}

Interviews were transcribed verbatim and data analysis was performed using qualitative content analysis [16]. Each interview was read repeatedly by each of the authors to gain an over all understanding of the content and an initial view of any common themes. The authors then discussed the transcribed interviews to identify any preliminary areas of interest. The transcriptions were subsequently re-read individually by the authors and divided into meaningful units based on the text's contents corresponding to the study aim. The units of meaning were condensed into concise text units by reducing the number of words while retaining the main substance. These concise text units were labelled with codes, where several different concise text units could have the same code. Codes with similar content formed subcategories. The subcategories were grouped into categories of related content. The subcategories and categories were created jointly by the authors.

\subsection{Rigour}

The variation in age, experience, and workplace of the chosen sample of RNs served to enhance credibility. In 


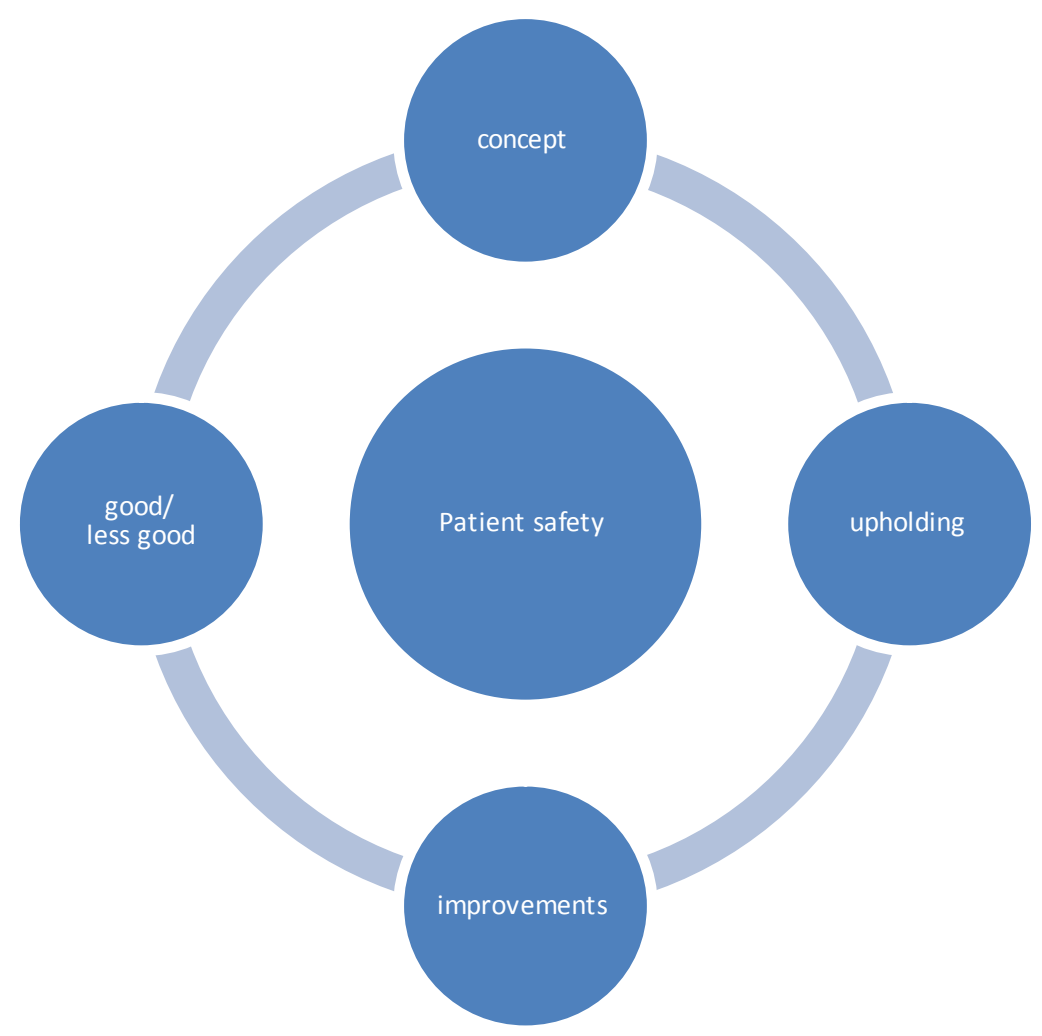

Figure 1. The palette that lay open as a common ground for questions and answers in all interviews.

presenting the findings, credibility has been strengthened by including representative quotations from the interviews [14]. Dependability has been furthered by clearly showing each step of the study. The authors sought confirmability, by asking the participants to clarify their statements during the interviews. Also, the authors strove to further strengthen confirmability by alternating between individual analysis and joint discussion, along with frequent double-checking of the transcripts throughout the analytic process [14] [16]. To promote transferability, the sample and setting have been described as clearly as possible without disclosing details that could identify the participants. The interview guide palette and follow up questions have also been presented.

\subsection{Ethical Considerations}

The study was performed based on the ethical principles stated in the WMA Declaration of Helsinki [17], with the exception of ethical approval, which was not required by national law since the study did not involve patients, physical examinations, nor in any way strive to influence the participants [18]. After receiving verbal and written information of the study being entirely voluntary, the participants signed a statement giving their informed consent. The audio-recordings and transcripts were stored confidentially. Findings were presented without disclosing the identity of any participant.

\section{Findings}

The findings consisted of two major categories. The first category, Support in regaining and maintaining health, comprised two subcategories pertaining to the individual needs and rights of the residents with SMI. The second category, Terms of daily living, consisted of five subcategories. It covered the overall areas of legislation and organization, along with professionals' knowledge, housing, and daily activity that constitute the external framework of CMHS. The findings indicated that the understanding of patient safety in CMHS for people with SMI was similar among all of the participating RNs. The individual needs of the person (resident) living in such CMHS were perceived as fundamental to patient safety. The availability of appropriate care at the right level, 
proper medication, and adequate support for a functioning daily life, were concrete elements in achieving patient safety. An overview of the findings is shown in Table 1.

\subsection{Support in Regaining and Maintaining Health}

This category described the need for professional care and support to regain and maintain health as a primary focus in patient safety. Two subcategories further described such needs as the need to be treated well by professionals and the ethical need for professionals to respect residents' self-determination and avoid coercion.

\subsubsection{Professional Treatment of Residents}

The way the RNs themselves and other professionals treated residents had a great influence on helping each resident find and use health-promoting resources of his/her own. The RNs found that residents often lacked an understanding of and the ability to use such resources in everyday life, and they stated that one important goal of patient safety was to help residents discover and use these resources. To provide safe patient care, the RNs considered it fundamental to relate to the resident as a unique person with individual needs beyond his/her mental disorder. Correct assessment of a resident's need for care depended on having well-founded knowledge about his/her normal state, which in turn called for a close relationship with the resident. To establish such relations, the RNs expressed a wish to spend more time in the CMHS. At the time this was not possible, mainly due to the administrative workload that was the RNs' responsibility and the large amount of time spent driving between the RNs' offices and the CMHS. Consequently, the RNs came to depend largely on reports from staff at the CMHS when making their professional decisions.

\subsubsection{The Line between Self-Determination and Coercion}

Being the resident's advocate was the RNs' starting point in providing safe patient care, and they considered each resident's right to self-determination as absolute and non-negotiable. However, that right also gave rise to difficult ethical dilemmas for the RNs. One example was the question of how safe patient care could be achieved when there was an obvious need for care but the resident would not give his/her consent:

How can you help somebody who doesn't want to be helped? Like when you have a decision that they need support in some area, they need that help. And they say no. But the fact that they say no doesn't mean we're not responsible for giving help. (N1)

One challenge met by the RNs was low compliance with pharmaceutical therapy on the part of residents. When a resident was unwilling to take his/her medication, the RNs described two options for handling the situation. The RN could both accept and respect the resident's "no" as self-determination or try to encourage the resident to follow the doctor's orders, thus taking the risk of transgressing the fine line between encouragement and coercion. In either case, patient safety could be endangered. Low compliance with prescribed psychiatric medication regimens could lead to deteriorating psychiatric health, causing a need for hospital care. When a resident would not consent and coercion was necessary if he/she was to receive the appropriate care, the RNs described the situation as a choice between deserting the resident and violating his/her right to self-determination:

Should she be allowed to sit there in her misery-because it was misery. Couldn't even, she wet herself, and we didn't even get to change her and, you know, then it's a matter of abuse... neglect. You, you have to choose.... (N5)

\subsection{Terms of Daily Living}

Communication in the large network surrounding the resident constituted an important element in maintaining patient safety. National laws and regulations, along with local routines and the benefits and problems of being part of a large public sector, all had implications for patient safety. Sufficient knowledge of psychiatric disabilities was important for everyone who came into professional contact with the residents. In accordance with the RNs' belief that patient safety emanates from the needs of the resident, everyday life settings played a role in creating safe patient care.

\subsubsection{Communication in the Resident's Network}

Well-functioning communication between the different parties in the resident's network was stressed as an 
Table 1. An overview of the findings.

\begin{tabular}{cc}
\hline \multicolumn{2}{c}{ Categories } \\
\hline Support in regaining and maintaining health & Terms of daily living \\
\hline Professional treatment of residents & Sub categories \\
The line between self-determination and coercion & Knowledge in the resident's network \\
Legislation and routines \\
Small field in a large organization \\
Care settings
\end{tabular}

important factor in patient safety. The RNs saw themselves as the hub of that network, feeling that they were expected to keep the various parties and professions influencing residents' daily life together. Advising other caregivers about the specific needs of a resident was one way of ensuring patient safety. The RNs talked about situations in which they had advised other caregivers about the specific needs of a resident in order to facilitate good treatment. The other side of that coin was respecting each resident's right to personal integrity and always obtaining consent before contacting other caregivers.

\subsubsection{Knowledge in the Resident's Network}

The RNs described knowledge from different perspectives. They saw gaps in knowledge about psychiatric needs among the different professions involved in mental health care and the CMHS. Improved knowledge on all levels in the organization was considered essential in improving patient safety. Concerning the political decision-makers and local officials who controlled CMHS, the RNs felt they lacked knowledge about the specific challenges and needs within the area of care. The RNs also felt those in control had inadequate knowledge about how disabilities could differ between residents with SMI and the mentally disabled:

People in the top think that you shouldn't present different disabilities, but a disability is a disability and sometimes it's like just whatever and I don't agree with that. (N5)

The RNs generally considered that staff at the CMHS was qualified to care for the residents. However, as it was not mandatory for enrolled nurses to have training in psychiatric care, the RNs saw a risk that lack of knowledge could lead to incorrect assessments. When the RNs were unable to spend as much time as they would like at the CMHS, the responsibility for detecting and assessing both psychiatric and physical problems was largely placed on the staff at CMHS. Psychiatric illness could cause residents to be unable to express what they felt, or even unable to recognize that something was physically wrong. In such cases, inadequate knowledge on the part of staff could pose a threat to patient safety. Another result of insufficient knowledge could be poor professional treatment. One important way of ensuring safe patient care was to hold regular staff meetings where the RNs could instruct and educate staff members.

\subsubsection{Legislation and Routines}

Regarding their everyday work, some of the RNs felt that the laws regulating CMHS were inflexible and made it difficult for them to employ simple and smooth solutions. One example of the lack of concordance between legislation and reality was when signature lists had to be kept separate from where the medicine was actually distributed. This caused a risk of mistakes in medication handling. Other aspects of national legislation that had a direct effect on residents were the laws regarding daily activity and level of support for the individual resident. The RNs felt these laws were often inadequate in meeting the needs of residents, because they made it difficult to provide occupations and activities that helped residents regain and maintain health.

Developing routines based on national legislation for everyday work in the CMHS, implementing these routines and improving them, were considered essential to patient safety. The RNs stated that most of the residents needed predictability in their everyday lives. Therefore, one aspect of developing well-functioning routines was establishing good habits and rules for the residents' life together. Encouraging staff to report adverse events was 
a hands-on way for the RNs to discover insufficient routines. Most of the adverse events that were reported concerned the handling of medicines. A few of the RNs had also received reports of inadequate treatment. The RNs considered it likely that adverse events were under-reported:

Staff probably don't want to report discrepancies, they don't see it as a way to deal with shortcomings. (N7)

As the reporting of adverse events was time consuming, some of the RNs said they sometimes refrained from filing reports on minor adverse events. Good routines in collaboration between staff and the RN were necessary to ensure that correct and relevant information was passed from staff to RN. Another area in which clearly set routines were important was in collaboration between different caregivers. Hospital care was provided by the county council, and some of the RNs considered the collaboration to be good. Others told of delays and misunderstandings in the reporting between caregivers and called for better, mutual routines. One example of insufficient routines for information exchange between caregivers was when a resident had been transferred from hospital to a sheltered housing facility. Lapses in information led to the resident becoming critically ill because he did not receive his medication for several days:

Somehow it's the patient who gets caught in the middle of all these dealings and conflicts about who should do what. (N4)

\subsubsection{Small Field in a Large Organization}

CMHS as a field was only a small part of the municipal organization compared to geriatric care, and this gave the RNs a sense of being overshadowed and forgotten in the organization. They felt they were often left to themselves in evaluating and reflecting on their work and considered this unsatisfactory from a patient safety perspective:

We try to get some collaboration going and I think we're moving in the right direction. We've felt a bit neglected. (N5)

Most of the RNs stated they had good support from psychiatrists and physicians in somatic health care, and that RNs in other fields were willing to help when asked questions. Having a superior who was familiar with the challenges of everyday work was highly appreciated.

\subsubsection{Care Settings}

All of the RNs talked about the residents' everyday environment as a vital part of patient safety. For their daily occupation, most residents were referred to workplaces intended for people with different kinds of disabilities. Not having access to activities specifically designed for psychiatric needs was considered potentially harmful to residents, as such a situation made it more difficult for them to regain and maintain health. Finding balance in the residents' daily activities was challenging, and underlined the need for professionals to have a great deal of knowledge about the residents. They also needed activities of some kind, and it was deemed important to find the right one.

Actually there isn't much to choose from, and it's also a matter of finding a balance because what they already have is often enough, like they maybe need help from staff with: taking care of their apartment and buying food and .... (N2)

Continuity in staffing and maintaining daily routines were said to be important in creating well-functioning CMHS, as the residents were often sensitive to change. The RNs described many of the buildings in which the facilities were located as dull, grey, and not well-suited to meeting residents' needs for both solitude and easily accessible company. According to the RNs, there was a risk to patient safety when a resident could not find a comfortable place to be in the facility, because this situation was stressful:

It's like a long corridor and then there are rooms and when they come out and maybe have been having problems with their thoughts and they walk right into each other. (N2)

All of the RNs identified poor nutrition as a risk factor for deteriorating health. Some of the CMHS offered residents cooked meals in common dining rooms, which was considered a good way to promote health by ensuring adequate nutrition: 
These patients are so ill that thinking they will take care of themselves and make their own food is utopian. (N1)

The RNs believed their professional competence and knowledge about the individual residents could be utilized better in finding ways to create the best possible patient group at each CMHS. There were examples of patients with the most severe illness being placed in the same facility, which led to problems that could probably have been avoided through better planning and by taking advantage of the RNs' knowledge about the residents.

\section{Discussion}

The main finding showed that quality of care was held high among the RNs to provide patient safety for people in CMHS. They saw the resident as an individual with a need for support and autonomy, an important factor given the environment surrounding the resident. The RNs did not refer to any standard guidelines regarding patient safety and defined the concept broadly, including variables that more generally reflect quality of care in medical nursing environments. They also expressed that supporting the resident to regain and maintain health, along with terms of daily living, was an overall necessity for ensuring patient safety among the residents.

WHO's definition of patient safety [1] does not include quality of care, yet this was what the RNs in the present study perceived as patient safety. WHO defines patient safety as absence of preventable harm to a patient during the process of healthcare. The RNs meant in line with Leapes' patient safety theory [2] that failure to provide, for example, an adequate daily occupation, or suitable support for a healthy lifestyle for a person with SMI constitutes a risk of harm.

Findings have shown that physical activity and some form of social contact is crucial in promoting the health of people with SMI [11] [19]. Even more important is that any activity undertaken is perceived as meaningful and an opportunity to develop closes social relations with others [19]. This is in line with our results. RNs talked about social inclusion among this group as more than general quality of care-they thought it was an issue of patient safety, i.e. providing adequate settings for daily living, a meaningful occupation, and encouragement to embrace a healthy lifestyle. In today's society this is a challenging task, where not only people with SMI are in need of a daily occupation and a healthy lifestyle.

The question of whether or not the residents are patients seems to be another stumbling block for the RNs and others, both in the health care organization and in society. Persons living in CMHS often suffer from long-term illness, but are not supposed to be considered patients because they live in their own apartments, although with round the clock access to staff. This needs clarification, because it in turn poses the question of whether the concept of patient safety is at all applicable if the residents are not patients. The specific needs of people with SMI call for legislation and guidelines on how to provide safe patient care to people afflicted by such illness.

In this study, RNs discussed the diversity and complexity of the area of CMHS. They perceived that this could constitute a risk to patient safety by entailing many different actors and possible lapses in professional treatment and communication concerning residents. The RNs perception of this is supported by Hunt et al. [20] saying adverse events and failure of care often depends on lapses in handover practices between staff surrounding the resident. In this study, it was hard for the RNs to get their voices heard about care quality issues. They felt that they were being left on their own since their sector of care was overshadowed by larger fields of care, i.e. people in CMHS are only a small part of the entire community health care system and that makes it harder to highlight the issues of care quality (patient safety). To work under such conditions has been reported as a threat to patient safety [5] [21] [22]. Another threat to patient safety may be untrained staff; in this study, only half of the RNs had a formal degree in mental health care and the enrolled nurses at the CMHS had nursing training at the upper secondary school level, though not always specialized in mental health care. This also seems to be the case in other countries; a literature review [11] showed that in the USA social workers and staff without mental health qualifications performs similar roles. Key ways to ensure safe mental health care are ongoing training in, for example, psychiatric assessment, breaking down the stigmas surrounding mental ill health, and provision of safe patient care by all involved staff [5].

There is no simple answer of how to enhance issues about quality of care, i.e. patient safety in the field of mental health in the community. The issues are multi-facetted and seem to be in line with the patient safety theory, which states that safe patient care is a matter of systemic dimensions [2]. However, close contact and collaboration between RNs and unit managers to strengthen living conditions for residents was one way suggested 
by the RNs in the present study. The necessity of defining the specifics of patient safety in CMHS and whether and how such specifics might differ from those in other sectors of health care can be a first step to emphasize issues of patient safety. With clarity on this, the RNs should have a common ground about the subject of patient safety and be in an optimal position to take charge of providing safe patient care. This is supported by early research showing that RNs are well equipped to take part in efforts to improve patient safety [13].

\section{Study Limitations}

A limitation of the study is the small sample of RNs, although the RNs were chosen purposively from a large geographic area. This raises the question of whether the present findings are representative of the population of RNs in the field as a whole. However, we feel that the variation in age, experience, and workplace of the sample of RNs served to enhance credibility. In presenting the findings, credibility has been strengthened through representative quotations from the interviews [14]. Arguably, the field of mental health care in the community is small. However, all over the world mental health care is being relocated from hospital settings to communities, making it a growing field of care.

\section{Conclusion}

The RNs in the present study discuss patient safety in the area of people in CMHS as involving a wide range of issues that in other areas of care are more often discussed in the broader sense of quality of care. Provision of care in CMHS raises the question of when a person becomes a patient, and of where the boundaries of patient safety lie. Determining the boundaries of patient safety in CMHS is the first step in establishing workable routines that ensure safe patient care.

\section{References}

[1] World Health Organization (2004) Patient Safety. http://www.who.int/patientsafety/about/en/

[2] Emanuel, L., Berwick, D., Conway, J., Combes, J., Hatlie, M., Leape, L., Reason, J., Schyve, P., Vincent, C. and Walton, M. (2008) What Exactly Is Patient Safety? In: Henriksen, K., Battles, J.B., Keyes, M.A. and Grady, M.L., Eds., Advances in Patient Safety: New Directions and Alternative Approaches (Vol. 1: Assessment), Agency for Healthcare Research and Quality, Rockville.

[3] Mitchell, P.H. (2008) Defining Patient Safety and Quality Care. In: Hughes, R.G., Ed., Patient Safety and Quality: An Evidence-Based Handbook for Nurses, AHRQ Publication No. 08-0043, Agency for Healthcare Research and Quality, Rockville, 1-5.

[4] Kanerva, A., Lammintakanen, J. and Kivinen, T. (2013) Patient Safety in Psychiatric Inpatient Care: A Literature Review. Journal of Psychiatric and Mental Health Nursing, 20, 541-548. http://dx.doi.org/10.1111/j.1365-2850.2012.01949.x

[5] Brickell, T.A. and McLean, C. (2011) Emerging Issues and Challenges for Improving Patient Safety in Mental Health: A Qualitative Analysis of Expert Perspectives. Journal of Patient Safety, 7, 39-44. http://dx.doi.org/10.1097/PTS.0b013e31820cd78e

[6] Briner, M. and Manser, T. (2013) Clinical Risk Management in Mental Health: A Qualitative Study of Main Risks and Related Organizational Management Practices. BMC Health Services Research, 13, 44. http://dx.doi.org/10.1186/1472-6963-13-44

[7] World Health Organization (2008) Integrating Mental Health into Primary Care: A Global Perspective. http://www.who.int/mental_health/policy/services/integratingmhintoprimarycare/en/

[8] World Health Organization (2011) Mental Health Atlas. http://whqlibdoc.who.int/publications/2011/9799241564359_eng.pdf

[9] Jenkins, R., Othieno, C., Okeyo, S., Aruwa, J., Kingora, J. and Jenkins, B. (2013) Health System Challenges to Integration of Mental Health Delivery in Primary Care in Kenya-Perspectives of Primary Care Health Workers. BMC Health Services Research, 13, 368. http://dx.doi.org/10.1186/1472-6963-13-368

[10] Hamden, A., Newton, R., McCauley-Elsom, K. and Cross, W. (2011) Is Deinstitutionalization Working in Our Community? International Journal of Mental Health Nursing, 20, 274-283. http://dx.doi.org/10.1111/j.1447-0349.2010.00726.x

[11] Happel, B., Hoey, W. and Gaskin, C.J. (2012) Community Mental Health Nurses, Caseloads, and Practices: A Literature Review. International Journal of Mental Health Nursing, 21, 131-137. http://dx.doi.org/10.1111/j.1447-0349.2011.00777.x 
[12] The National Board of Health and Welfare (2014) National Planning Support in 2014—Supply and Demand of Certain Groups of Staff in Healthcare and Dental Care. http://www.socialstyrelsen.se/publikationer2014/2014-1-32

[13] Mwachofi, A., Walston, S.L. and Al-Omar, B.A. (2011) Factors Affecting Nurses’ Perceptions of Patient Safety. International Journal of Health Care Quality Assurance, 24, 274-283. http://dx.doi.org/10.1108/09526861111125589

[14] Polit, D.F. and Beck, C.T. (2012) Nursing Research: Generating and Assessing Evidence for Nursing Practice. Lippincott Williams and Wilkins, Philadelphia.

[15] Swedish Code of Statutes, SFS (2010) Swedish Act on Patient Safety. http://www.riksdagen.se/sv/Dokument-Lagar/Lagar/Svenskforfattningssamling/Patientsakerhetslag-2010659_sfs-2010$\underline{659 /}$

[16] Graneheim, U.H. and Lundman, B. (2004) Qualitative Content Analysis in Nursing Research: Concepts, Procedures and Measures to Achieve Trustworthiness. Nurse Education Today, 24, 105-112. http://dx.doi.org/10.1016/j.nedt.2003.10.001

[17] Declaration of Helsinki (2013) World Medical Association. http://www.wma.net/en/20activities/10ethics/10helsinki/index.html

[18] Swedish Ethical Review Act (2003) SFS. Vetting the Ethics of Research Involving Humans. http://www.epn.se/en/start/startpage/

[19] Argentzell, E., Leufstadius, C. and Eklund, M. (2014) Social Interaction among People with Psychiatric DisabilitiesDoes Attending a Day Center Matter? International Journal of Social Psychiatry, 60, 519-527. http://dx.doi.org/10.1177/0020764013502318

[20] Hunt, G.E., Marsden, R. and O’Connor, N. (2012) Clinical Handover in Acute Psychiatric and Community Mental Health Settings. Journal of Psychiatric and Mental Health Nursing, 19, 310-318. http://dx.doi.org/10.1111/j.1365-2850.2011.01793.x

[21] Magnusson, A., Hogberg, T., Lutzen, K. and Severinsson, E. (2004) Swedish Mental Health Nurses’ Responsibility in Supervised Community Care of Persons with Long-Term Mental Illness. Nursing \& Health Sciences, 6, 19-27. http://dx.doi.org/10.1111/j.1442-2018.2003.00171.x

[22] Kristiansen, L., Hellzen, O. and Asplund, K. (2010) Left Alone-Swedish Nurses’ and Mental Health Workers’ Experiences of Being Care Providers in a Social Psychiatric Dwelling Context in the Post-Health-Care-Restructuring Era. A Focus-Group Interview Study. Scandinavian Journal of Caring Sciences, 24, 427-435. http://dx.doi.org/10.1111/j.1471-6712.2009.00732.x 